CERN-PH-TH/2005-209, RI57/05, hep-th/0511061

\title{
Multitrace Deformations of Vector and Adjoint Theories and their Holographic Duals
}

\author{
S. Elitzur ${ }^{a}$, A. Giveon ${ }^{a}$, M. Porrati ${ }^{b, c}$ and E. Rabinovici ${ }^{a, d}$ \\ ${ }^{a}$ Racah Institute of Physics \\ the Hebrew University \\ Jerusalem, IL 91904, ISRAEL \\ ${ }^{b}$ Department of Physics \\ New York University \\ 4 Washington Pl. \\ New York, NY 10003, USA \\ ${ }^{c}$ Scuola Normale Superiore \\ Piazza dei Cavalieri 7 \\ I-56126, Pisa, ITALY \\ ${ }^{d}$ PH Department, TH Unit \\ CERN \\ CH 1211 Geneva 23, SWITZERLAND
}

\begin{abstract}
We present general methods to study the effect of multitrace deformations in conformal theories admitting holographic duals in Anti de Sitter space. In particular, we analyse the case that these deformations introduce an instability both in the bulk AdS space and in the boundary CFT. We also argue that multitrace deformations of the $O(N)$ linear sigma model in three dimensions correspond to nontrivial time-dependent backgrounds in certain theories of infinitely many interacting massless fields on $A d S_{4}$, proposed years ago by Fradkin and Vasiliev. We point out that the phase diagram of a truly marginal large- $N$ deformation has an infrared limit in which only an $O(N)$ singlet field survives. We draw from this case lessons on the full string-theoretical interpretation of instabilities of the dual boundary theory and exhibit a toy model that resolves the instability of the $O(N)$ model, generated by a marginal multitrace deformation. The resolution suggests that the instability may not survive in an appropriate UV completion of the CFT.
\end{abstract}




\section{Introduction}

Three dimensional Conformal Field Theories (CFTs) are of special interest as they may well define macroscopic four dimensional local theories of gravity. In addition, if the boundary theory consists of scalar fields $\phi$ in the fundamental representation of a global $O(N)$ symmetry, much is known about the system, in particular in its large $N$ limit. The emerging structure is quite rich and includes isolated fixed points as well as a line of fixed points and flat and unstable directions. It is not yet known if every CFT defined on some boundary admits a corresponding local bulk theory. In the absence of that knowledge one can still identify several interesting features that such a corresponding bulk theory would have, if it exists.

One such case may correspond to a limit of string theory on $A d S_{4}$ in which there exists just one flat Regge trajectory with massless particles of even spin. It was suggested in [1] (see 2] for a recent review) that such a theory of massless high spin particles exists in an $A d S_{4}$ background. The exact quantum description of such a system is still lacking, but one could consider instead its CFT dual, which was conjectured in [3] to be a three dimensional theory of vector particles, $\phi$, with a global $O(N)$ symmetry. This was based on the identification of $O(N)$-singlet, conserved currents in the boundary theory with the correct quantum numbers to correspond to massless even spin particles in the bulk. The identification (or elimination) of the many conserved non-singlet currents was not resolved.

In 3] two fixed points of such three dimensional theories of scalars were discussed: the trivial fixed point and the non-trivial fixed point, which are known to occur in the presence of a relevant perturbation of the form $g_{4}\left(\phi^{2}\right)^{2}$ in the large $N$ limit. A dual mapping between these two versions of the same bulk theory was suggested as well. It was also shown [4 that the boundary theory at the non-trivial fixed point has all the features needed to describe a Higgs phenomenon in the bulk. In particular, $1 / N$ corrections allow all particles with spin larger than two to obtain a mass. We review these results in section 2. Classically, the scalar theory has also a marginal deformation; it is generated by the operator $\left(\phi^{2}\right)^{3}$. For any finite positive value of the deformation parameter $g_{6}$ the operator is quantum mechanically irrelevant and this infrared free theory is only well defined for zero renormalized value of $g_{6}$. Namely, for any finite value of $g_{6}$ the theory can only be defined in the presence of a cutoff; it contains only particles whose mass is of the order of the cutoff and thus is afflicted with all the bad properties of such nonrenormalizable theories. In the strict large $N$ limit a window opens up, and the theory becomes well defined for a finite range of $g_{6}: g_{6} \leq g_{c}[5]$. The behaviour of the theory at the critical value

of $g_{6}$ and beyond are of interest. For example, at the critical value the scale invariance can be spontaneously broken and a mass scale can be generated. The effective infrared limit contains 
now only conserved flavor-singlet currents.

In section 3 we review and discuss the properties of these theories and their possible consequences for the bulk duals. This will touch both upon the dual tensionless phase of strings and on possible quantum resolution of classical singularities. In section 4 we establish a rather general effective field theory approach to usefully deal with multi trace deformation. In section 5 we leave the safe realm of scalar vector particles which have a well defined boundary theory and venture into the more shady bulk description of a system which has interesting bulk properties, including classical space-like singularities and a strongly interacting three dimensional CFT dual, namely the IR limit of a super Yang-Mills theory with 16 supercharges. This theory too can be deformed by a marginal operator of the form $\left(\operatorname{Tr} \phi^{2}\right)^{3}$, where $\phi$ denotes appropriate scalars of the theory. There, we also discuss the issue and significance of possible instabilities and their resolution in the boundary theory.

Finally, in section 6, we expand on the meaning of such instabilities in string theory ${ }^{1}$. In particular, we discuss when string theory is expected to resolve singular bulk configurations, and when it is not. We indicate that the instabilities in the dual theory relate essentially to bulk configurations whose singularities are of a nature that does not need to be resolved by string theory in a near-horizon limit.

\section{The $O(N)$ Vector Model and its Marginal Deformation}

Let us now review some known facts about the three dimensional theory once a classically marginal operator, $\left(\phi^{2}\right)^{3}$, is added [5]. For any finite value of $N$, the coupling $g_{6}$ of this operator is infrared free quantum mechanically, as the marginal operator gets a positive anomalous dimension already at one loop. This implies that the theory is only well defined for zero value of the coupling of this operator. In the presence of a cutoff interacting particles have mass of the order of the cutoff. At its tri-critical point the $O(N)$ model in three dimensions is described by the Lagrangian,

$$
\mathcal{L}=\frac{1}{2} \partial_{\mu} \phi \cdot \partial^{\mu} \phi+\frac{1}{6 N^{2}} g_{6}\left(\phi^{2}\right)^{3}
$$

where the fields $\phi$ are in the vector representation of $O(N)$. In the limit, $N \rightarrow \infty$,

$$
\beta_{g_{6}}=0
$$

$1 / N$ corrections break conformality. In the large $N$ limit, then, $g_{6}$ is a modulus. There is no spontaneous breaking of the $O(N)$ symmetry and it is instructive to write the effective potential

\footnotetext{
${ }^{1}$ Other consequences of instabilities in the presence of a mass gap were studied in 6 .
} 
in terms of an $O(N)$ invariant field,

$$
\sigma=\phi^{2}
$$

notice that the renormalized $\sigma$ is non-positive! [5]. The effective potential is:

$$
V(\sigma)=f\left(g_{6}\right)|\sigma|^{3}
$$

where:

$$
f\left(g_{6}\right)=g_{c}-g_{6}
$$

with

$$
g_{c}=(4 \pi)^{2}
$$

The system has various phases. For values of $g_{6}$ smaller than $g_{c}$, i.e. when $f\left(g_{6}\right)$ is positive, the system consists of $N$ massless non-interacting $\phi$ particles. These particles do not interact in the infinite $N$ limit; thus, correlation functions do not depend on $g_{6}$ and all the conserved currents identified by [3] do not aquire anomalous dimensions along that direction. The corresponding high spin particles in the bulk are thus still massless.

For the special value $g_{6}=g_{c}, f\left(g_{6}\right)$ vanishes and a flat direction in $\sigma$ opens up: the expectation value of $\sigma$ becomes a modulus. For a zero value of this expectation value, the theory continues to consist of $N$ massless $\phi$ fields. For any non-zero value of the expectation value the system has $N$ massive $\phi$ particles. All have the same mass due to the unbroken $O(N)$ symmetry. Scale invariance is broken spontaneously so the vacuum energy still vanishes. The Goldstone boson associated with the spontaneous breaking of scale invariance, the dilaton, is massless and identified as the $O(N)$ singlet field $\delta \sigma \equiv \sigma-\langle\sigma\rangle$. All the particles are non-interacting in the infinite $N$ limit. This theory is not conformal: in the infrared limit, it flows to another theory containing a single, massless, $O(N)$-singlet particle. From this singlet, one can construct even-spin conserved currents which are now however all $O(N)$ singlets. For larger values of $g_{6}$ the exact potential is unbounded from below. The system is unstable (in the supersymmetric case the potential is bounded from below and the larger $g_{6}$ structure is similar to the smaller $g_{6}$ structure [7]). Actually this instability is an artifact of the dimensional regularization used above, which does not respect the positivity of the renormalized field $\sigma$. Still it will be useful when we discuss instabilities. In any case a more careful analysis [5] shows that the apparent instability reflects the inability to define a renormalizable interacting theory, all masses are of the order of the cutoff and there is no mechanism to scale them down to low mass values. In other words, the theory depends strongly on its UV completion. This is summarized in Table 1. There, S.B. denotes spontaneous symmetry breaking of scale invariance and $V$ is the vacuum 
Table 1: Marginal Perturbations of the $O(N)$ Model

$\begin{array}{ccccc}f\left(g_{6}\right) & |\langle\sigma\rangle| & \text { S.B. } & \text { masses } & V \\ f\left(g_{6}\right)>0 & 0 & \text { No } & 0 & 0 \\ f\left(g_{6}\right)=0 & 0 & \text { No } & 0 & 0 \\ f\left(g_{6}\right)=0 & \neq 0 & \text { Yes } & \text { Massless dilaton, } N & 0 \\ f\left(g_{6}\right)<0 & \infty & \text { Yes, } & \begin{array}{c}\text { particles of equal mass } \\ \text { Tachyons or masses } \\ \text { but ill defined }\end{array} & -\infty \\ & & \text { of order the cutoff } & \end{array}$

energy. For $f\left(g_{6}\right)<0$ the theory is unstable. Note that the vacuum energy always vanishes whenever the theory is well-defined.

When $\langle\sigma\rangle \neq 0$ and the scale invariance is spontaneously broken, one can write down the effective theory for energy scales below $\langle\sigma\rangle$, and integrate out the degrees of freedom above that scale. The vacuum energy remains zero, however, and is not proportional to $\langle\sigma\rangle^{3}$ as might be expected naively (see [8] and references therein).

For completeness and for potential future use we note that by adding more vector fields one has also phases in which the internal global $O(N)$ symmetry is spontaneously broken.

An example is the $O(N) \times O(N)$ model [9] with two fields in the vector representation of $O(N)$, with Lagrangian:

$$
\begin{aligned}
\mathcal{L}= & \partial_{\mu} \boldsymbol{\phi}_{1} \cdot \partial^{\mu} \boldsymbol{\phi}_{1}+\partial_{\mu} \boldsymbol{\phi}_{2} \cdot \partial^{\mu} \boldsymbol{\phi}_{2}+\lambda_{6,0}\left(\boldsymbol{\phi}_{1}^{2}\right)^{3}+ \\
& \lambda_{4,2}\left(\boldsymbol{\phi}_{1}^{2}\right)^{2}\left(\boldsymbol{\phi}_{2}^{2}\right)+\lambda_{2,4}\left(\boldsymbol{\phi}_{1}^{2}\right)\left(\boldsymbol{\phi}_{2}^{2}\right)^{2}+\lambda_{0,6}\left(\boldsymbol{\phi}_{2}^{2}\right)^{3}
\end{aligned}
$$

Again the $\beta$ functions vanish in the strict $N \rightarrow \infty$ limit. There are now two possible scales, one associated with the breakdown of a global symmetry and another with the breakdown of scale invariance. The possibilities are summarized by the table below:

$$
\begin{array}{cccccc}
O(N) & O(N) & \text { scale } & \text { massless } & \text { massive } & V \\
+ & + & + & \text { all } & \text { none } & 0 \\
- & + & - & (N-1) \pi^{\prime} s, D & N, \sigma & 0 \\
+ & - & - & (N-1) \pi^{\prime} s, D & N, \sigma & 0 \\
- & - & - & 2(N-1) \pi^{\prime} s, D & \sigma & 0
\end{array}
$$

Again, in all cases the vacuum energy vanishes. Assume a hierarchy of scales where the scale invariance is broken at a scale much above the scale at which the $O(N)$ symmetries are broken. One would have argued that one would have had a low energy effective Lagrangian for the massless pions and dilaton with a vacuum energy given by the scale at which the global symmetry is broken. This is not true, the vacuum energy remains zero. This system has a critical surface, on one patch the deep infrared theory contains only one massless particle: an $O(N) \times O(N)$ 
singlet. For the other patches the deep infrared theory is described by $\mathcal{O}(N)$ massless particles, most of which are not singlets.

\section{The $A d S_{4}$ Dual of the Deformed $O(N)$ Model}

The detailed information on the boundary theory is not matched by as much information about the bulk theory. As we mentioned earlier, [3] have conjectured that the subsector of the theory defined by correlators of $O(N)$-singlet conserved currents is dual to one of the theories studied by Vasiliev. These theories can be defined only in $A d S$ spaces and contain an infinite number of massless particles of any spin. In a sense, these theories contain one flat "Regge trajectory." No statement was made about nonsinglet currents ${ }^{2}$.

In the absence of a clear identification we propose here a modified correspondence. There are indication that the bulk theory corresponding to the trivial Gaussian fixed point and its dual fixed point indeed contains a flat Regge trajectory but it could - indeed it must [10] contain many such trajectories perhaps as many as $N^{2}$. These would include the non-singlet massless fields as well as massive fields. We call the resulting bulk theory a Fradkin-Vasiliev system. The addition of a weakly coupled marginal operator will not change the geometry. However once the coupling $g_{6}$ reaches the critical value the situation changes. In particular the geometry corresponding to the infrared limit in the presence of a non zero expectation value for $\sigma$ would correspond to the pure Vasiliev system. As the boundary theory contains a single field we expect the bulk theory to contain a well defined geometry but with a curvature larger than in the initial $A d S_{4}$. Specifically, we believe that the bulk description of the CFT at $g_{6}=g_{c}$ goes as follows. The VEV $\langle\sigma\rangle \neq 0$ introduces a mass scale. At energies $E \gg|\langle\sigma\rangle|$, the boundary theory has $\mathcal{O}\left(N^{2}\right)$ conserved currents. At $E \ll|\langle\sigma\rangle|$, the effective low-energy theory contains only the field $\sigma$, so only currents built out of it survive.

Moreover, the boundary theory contains a-priori two dimensionless parameters, $N$ and $g_{6}$. In addition it may contain a length scale $R$ associated with the spatial part of the world volume. In the case of a four dimensional boundary, the corresponding parameters are $g_{Y M}^{2}, N$ and $R$. The difference is that unlike the three dimensional system, in the large $N$ limit, the bosonic system does not depend on the value of $g_{6}$ as long as it is smaller than $g_{c}$; the behavior is different for $g_{6}=g_{c}$, as described above. In the supersymmetric system at the critical point $g_{6}=g_{c}$, scale invariance can also break spontaneously. In this case, the far-infrared effective theory consists

\footnotetext{
${ }^{2}$ Remarkably though, even at $\mathcal{O}(1 / N)$ one can consistently restrict the theory to the singlet sector, and interpret the anomalous dimensions of the currents in terms of a bulk symmetry breaking mechanism in the Vasiliev theory [4].
} 
of two free massless particles, the dilaton and the dilatino. The boundary theory must thus contain appropriate additional conserved currents. The corresponding bulk theory will consist of additional higher spin massless states organized in supermultiplets. Such systems make sense also for larger values of the coupling constant, $g_{6}>g_{c}$. Once again there is no $g_{6}$ dependence there. This may indicate that unlike the four dimensional case where the bulk theory depends on three parameters, for example, $g_{s}, N$, and $R$, in the three dimensional boundary case the bulk theory depends on one parameter less. A possible consequence of this is that the bulk theory has no small curvature limit at all. In the bulk, this behavior could be reproduced if the $4 \mathrm{~d}$ space approaches $A d S_{4}$ with a radius of curvature of order one near the boundary ${ }^{3}$, but deep into the bulk it deviates from it and then eventually asymptotes to another $A d S_{4}$ space, with an even smaller radius of curvature. Perhaps even as small as $\mathcal{O}(1 / N)$. Somewhat similar phenomena exist for $N=4 \mathrm{SYM}$ [19]. The width of the bulk transition region should be proportional to $1 /\langle\sigma\rangle$.

What about the large $g_{6}$ behavior? The bosonic boundary theory is ill defined. In particular, a configuration on the boundary would decay in a finite time. In the bulk this means that well defined initial data evolve into a naked singularity which reaches the boundary in a finite time. This is an example of a bulk singularity that is not resolved by the boundary theory. In fact we think this could be a little misleading. The true meaning of the instability is clear in the presence of the cutoff: the boundary operator at finite $N$ is UV relevant, so it is badly behaved in the UV. In the bulk dual, a UV relevant operator corresponds to a perturbation of $A d S_{4}$ that diverges near the boundary ${ }^{4}$. This cannot be a localized, finite-energy initial state, since space is changed infinitely far away from each interior point. So, the fact that this infinite-energy configuration evolves into a singularity is not really surprising, and it may not contradict the hope that every finite-energy, localized initial configuration evolves either smoothly or towards a singularity that can be resolved by the boundary CFT.

We shall argue that the same phenomenon occurs in the case of a more complicated boundary CFT, namely that describing the $A d S_{4} \times S_{7}$ compactification of d=11 supergravity/M theory. That example was considered by Hertog and Horowitz [11, 12, 13]. The boundary theory in that case is more complicated, since it is the infraded limit of a 3d SYM theory with 16 supercharges. The theory still bears some similarity with the $O(N)$ model, since it also possesses composite scalar operators of dimension one. We next review and elaborate on the tools needed to monitor

\footnotetext{
${ }^{3}$ The theory possesses a fundamental length defined as the scale at which it becomes essentially nonlocal.

${ }^{4}$ Technically, this is true only for "single-trace" deformations, that is, for deformations corresponding to one particle states in the bulk. On the other hand, at finite $N$, multitrace operators mix with single trace ones. In the bulk the dual phenomenon is that in an interacting theory there is no sharp distinction between multi-particle and one-particle states.
} 
the modifications induced in the bulk theory by various changes of the boundary theory.

\section{Multitrace Deformations and their Use}

In this section we rederive some known results about multitrace deformations using a unifrom language, that is well suited to study both vector and adjoint theories. Let us consider a $\mathrm{CFT}_{d}$ with $A d S_{d+1}$ dual. An operator $\mathcal{O}$ of dimension $\Delta$ (scalar, for simplicity) is associated to a bulk field $\Sigma$. The AdS line element is

$$
d s^{2}=\frac{L^{2}}{z^{2}}\left(d z^{2}+d x^{m} d x_{m}\right)
$$

and the boundary condition for the scalar is

$$
\Sigma \sim \alpha z^{d-\Delta}+\beta z^{\Delta}
$$

$\alpha$ is identified with the source for $\mathcal{O}$, and $\beta$ is (proportional to) the $\operatorname{VEV}$ of $\mathcal{O}$ :

$$
\alpha=J, \quad \beta=d\langle\mathcal{O}\rangle_{J}
$$

The generating functional of the connected Green functions for $\mathcal{O}, W[J]$, is given by an action on $A d S_{d+1}$, which depends on the field $\Sigma$ as well as other fields, such as the metric fluctuation etc. In the case of $O(N)$ models the explicit form of this action is still a mystery, but the boundary theory is well known. In the case of the theory living on M-theory branes, AdS/CFT leads us to the action of $N=1$ supergravity in 11 d compactified on $A d S_{4} \times S_{7}$. The action must be computed on-shell with boundary condition $\alpha=J$ for $\Sigma$. Explictly

$$
\left.S[\Sigma, \ldots]\right|_{\text {on-shell }} \equiv S[\alpha],\left.\quad S[\alpha]\right|_{\alpha=J}=W[J]
$$

Notice that $S$ depends on $\alpha$ only, because $\beta$ becomes a fixed function of $\alpha$ by requiring regularity inside $A d S_{d+1}$. Thanks to the standard identity

$$
\langle\mathcal{O}\rangle_{J}=\frac{\delta W[J]}{\delta J}
$$

we can also write

$$
\beta=d \frac{\delta S[\alpha]}{\delta \alpha}
$$

Define now $\sigma=\langle\mathcal{O}\rangle_{J}=\beta / d$. In this formula, the $\mathrm{VEV}$ of $\mathcal{O}$ is $\beta / d$ because of the relation between $\beta$ and the VEV of $\mathcal{O}$ given in Eq. (12) 
Call $F[\sigma]$ the Legendre transform of $W[J]$. Then we have the standard identities

$$
F[\sigma]=W[J]-J \sigma, \quad \sigma=\frac{\delta W[J]}{\delta J}, \quad J=-\frac{\delta F[\sigma]}{\delta \sigma} .
$$

By construction, $F[\sigma]$ is the effective action of the undeformed CFT; i.e. the generator of 1PI correlators for the operator $\mathcal{O}$. One can add boundary terms to the bulk action $S$; by choosing them judiciously, and by expressing $S$ in function of the coefficient $\beta$, one has

$$
\left.S[\beta]\right|_{\beta=\sigma}=F[\sigma] .
$$

Now, let us deform the CFT by adding a multi-trace deformation $V(\mathcal{O})+J \mathcal{O}$. The effect of multitrace deformations within the AdS/CFT duality was derived in [14, 15]. It amounts to setting

$$
\alpha=J+V^{\prime}(\beta / d) .
$$

Equations (1518) can be written as

$$
\alpha=J+V^{\prime}(\sigma), \quad \alpha=-\frac{\delta F[\sigma]}{\delta \sigma} .
$$

So, $J=-V^{\prime}(\sigma)-\delta F[\sigma] / \delta \sigma$. This equation tells us that the generating functional of the deformed theory is simply:

$$
F_{V}[\sigma]=F[\sigma]+\int d^{d} x V[\sigma] .
$$

This result is independent of the AdS/CFT correspondence and holds for any theory admitting a large $N$ expansion. Let us make explicit the $N$ dependence of our formulas (in the adjoint case, to be concrete). Define $\mathcal{O}$ such that its VEV is $\mathcal{O}\left(N^{0}\right)$, i.e. finite in the large- $N$ limit. $W[J]$ is $\mathcal{O}\left(N^{2}\right)$ so we define $W[J]=N^{2} w[J]$. The functional integral over CFT fields $\phi$ gives

$$
e^{-N^{2} w[J]}=\int[d \phi] e^{-S-\int d^{d} x N^{2} J \mathcal{O}} .
$$

Add a multi-trace deformation $V(\mathcal{O})=N^{2} v(\mathcal{O})$ using the method of [16], and define $F[\sigma]=$ $N^{2} f[\sigma]$. Then:

$$
\begin{aligned}
e^{-N^{2} f_{V}[\sigma]} & =\int[d \phi][d J] e^{-S-\int d^{d} x N^{2}[J \mathcal{O}-J \sigma+v(\sigma)]} \\
=\int[d J] e^{-N^{2}\left\{w[J]+\int d^{d} x[-J \sigma+v(\sigma)]\right\}} & =e^{-N^{2}\left\{f[\sigma]+\int d^{d} x v(\sigma)\right\}} .
\end{aligned}
$$

The last equality holds because in the large $N$ limit the functional integral reduces to its saddlepoint approximation.

Equation (201) - or, equivalently Eq. (221) - contains in a compact form all results on multitrace deformations known in the literature. A similar formula was proposed in ref. [17. Now, let us recover a few known results, and conclude with a few comments. 
1. In the $3 \mathrm{~d} O(N)$ model, $F[\sigma]=-g_{c} \sigma^{3}, \sigma<0[5]$. Then, the deformation $V(\sigma)=g_{6} \sigma^{3}$ is marginal for all $\lambda$, and the potential is bounded below for $g_{6}<g_{c}$, as previously announced. At $g_{6}=g_{c}, \sigma$ becomes a modulus.

2. Let us add a quadratic perturbation, $V=\lambda \sigma^{2}$, and assume $\Delta<d / 2$, as in [14, 18]. According to our Eq. (20), the effective potential $F$ is

$$
F_{V}[\sigma]=\int d^{d} k \tilde{\sigma}(-k)\left[k^{d-2 \Delta}+\lambda\right] \tilde{\sigma}(k)+O\left(\sigma^{3}\right)
$$

Here the tilde denotes the Fourier transform. In the UV, $k^{2 \Delta-d} \gg \lambda$ and the connected two-point function of $\mathcal{O}$ is

$$
\langle\tilde{\mathcal{O}}(k) \mathcal{O}(0)\rangle \sim k^{2 \Delta-d}
$$

This equation says that the UV dimension of $\mathcal{O}$ is $\Delta$. In the IR, $k^{d-2 \Delta} \ll \lambda$, so that

$$
\langle\tilde{\mathcal{O}}(k) \mathcal{O}(0)\rangle \sim \frac{1}{k^{d-2 \Delta}+\lambda}=\frac{1}{\lambda}-\frac{k^{d-2 \Delta}}{\lambda^{2}}+O\left(\lambda^{-3}\right) .
$$

The first term in the expansion is just a contact term; the second says that the IR dimension of $\mathcal{O}$ is $d-\Delta(\operatorname{cfr}[14,18])$. Notice that the IR theory is nontrivial because $k^{d-2 \Delta}$ is (generically) nonlocal. Of course, a standard kinetic term for $\sigma$, proportional to $k^{2}$ would give a trivial IR fixed point. When $\Delta$ is an integer, one has to remember that the $\sigma$ kinetic term is still nonlocal because it is of the form $k^{2 \Delta} \log k^{2}$.

3. In [14, Witten gives an example of one-loop flow induced by a double-trace perturbation. We can recover that behavior thanks to our Eq. (20).

Choose $\Delta=d / 2$. Then we have, instead of Eq. (23),

$$
F_{V}[\sigma]=\int d^{d} k \tilde{\sigma}(-k)\left[\frac{1}{\log \left(k^{2} / \mu^{2}\right)}+\lambda\right] \tilde{\sigma}(k)+O\left(\sigma^{3}\right) .
$$

Next, renormalise the coupling by demanding that the kinetic term vanishes at a constant $k^{2}=\Lambda^{2}$, independent of $\mu$. This defines the running of $\lambda$ by the equation

$$
\frac{1}{\lambda(\mu)}=-\log \left(\Lambda^{2} / \mu^{2}\right)
$$

Up to obvious manipulations, this is the same as Witten's Eq. (4.9) 14.

4. Notice that it is always possible to create flat directions in $\sigma$ by choosing $\sigma$ constant and deforming with $V(\sigma)=-F(\sigma)$. 
5. To preserve conformal invariance, we need

$$
V\left(\lambda^{\Delta} \sigma\right)=\lambda^{d} V(\sigma) .
$$

In three dimensions this gives us back the cubic potential for deformations of conformal weight one.

Consider now again the perturbation $V=\left(g_{6} / 3\right) \sigma^{3}$ in the $O(N)$ model. Here $d=3, \Delta=1$. In our conventions, the entire action is multiplied by $N$ :

$$
S=N \int d^{3} x\left[\partial_{\mu} \phi \partial^{\mu} \phi+J \phi^{2}-J \sigma+V(\sigma)\right] .
$$

When $g_{6}=g_{c}$, a one-dimensional moduli space appears. The modulus is the $\operatorname{VEV}\left\langle\phi^{2}\right\rangle=\sigma$. The equation relating $\sigma$ to $J$ is

$$
J=\left(g_{c}-g_{6}\right) \sigma^{2} .
$$

By keeping the physical mass (i.e. $J$ ) constant, we get the RG trajectory of ref. [5, upon identifying $|\sigma|$ with the mass scale $\mu$ :

$$
0=-\frac{d g_{6}}{d \mu} \mu+2\left(g_{c}-g_{6}\right) .
$$

We can also easily find the existence of a Goldstone pole $1 / p^{2}$ for the composite state $\phi^{2}$. Indeed, when $J$ is nonzero $W[J]$ can be expanded in powers of derivatives as (we specialize our results to $d=3, \Delta=1$ )

$$
W[J]=N \int d^{3} x\left[w(J)+G(J) \partial_{\mu} J \partial^{\mu} J+\ldots .\right] .
$$

Here ... stands for higher-derivative terms. Straightforward dimensional analysis and Eqs. (16) give

$$
w(J)=-\frac{2}{3} \lambda_{c}^{-1 / 2} J^{3 / 2}, \quad G(J) \propto J^{-3 / 2} .
$$

The effective action $F[\sigma]$ also admits a derivative expansion, whenever $\sigma \neq 0$ :

$$
F[\sigma]=\int d^{3} x\left[-\left(\lambda_{c} / 3\right) \sigma^{3}+\tilde{G}(\sigma) \partial_{\mu} \sigma \partial^{\mu} \sigma+\ldots .\right] .
$$

The kinetic term $\tilde{G}(\sigma)$ can be obtained by explicitly performing the Legendre transformation or by dimensional analysis. In either ways we get $\tilde{G}(\sigma) \propto \sigma^{-1}$.

The perturbed generating functional $F_{V}[\sigma]$ is given by Eq. (20), so, at $g_{6}=g_{c}, F_{V}[\sigma]$ exhibits a $1 / p^{2}$ Goldstone pole outside the origin of the moduli space

$$
F_{V}[\sigma]=\text { constant } \times N \int d^{3} x \sigma^{-1} \partial_{\mu} \sigma \partial^{\mu} \sigma+\ldots \ldots
$$


Of course, this expansion makes sense only when $|\partial \sigma| \ll \sigma^{2}$.

Let us examine next the conserved currents in the perturbed $O(N)$ model. In the free $O(N)$ model there exist an infinite number of them:

$$
J_{\mu_{1} \ldots \mu_{n}}(x)=\phi \stackrel{\leftrightarrow}{\partial}_{\mu_{1}} \ldots \stackrel{\leftrightarrow}{\partial}_{\mu_{n}} \phi
$$

When $g_{6}=g_{c}$ and $\sigma=\left\langle\phi^{2}\right\rangle \neq 0, \sigma$ becomes the interpolating field of a zero-mass Goldstone particle:

$$
\left\langle p\left|\phi^{2}(x)\right| 0\right\rangle=F_{\sigma} \exp \left(i p_{\mu} x^{\mu}\right), \quad p^{2}=0 .
$$

The constant $F_{\sigma}$ is the analog of the pion coupling strength. Now, at leading order in $1 / N, \sigma$, and therefore $J$, are nonzero constants. So, the correlators

$$
\left\langle 0\left|J_{\mu_{1} \ldots \mu_{n}}(x) \phi^{2}(y)\right| 0\right\rangle, \quad\left\langle 0\left|J_{\mu_{1} \ldots \mu_{n}}(x) \phi^{2}(y) \phi^{2}(z)\right| 0\right\rangle,
$$

are one-loop integrals made of the free, massive propagators of the fields $\phi^{a}, a=1, \ldots ., N$. So, they are nonzero and localized. More precisely, they behave as, e.g.

$$
\left\langle 0\left|J_{\mu_{1} \ldots \mu_{n}}(x) \phi^{2}(y)\right| 0\right\rangle \sim \exp \left(-J^{1 / 2}|x-y|\right), \quad \text { for }|x-y| \gg J^{-1 / 2} .
$$

Because of Eq. (37), this means also that the matrix elements of currents between one- and two-Goldstone boson states are nonzero:

$$
\left\langle 0\left|J_{\mu_{1} \ldots \mu_{n}}(x)\right| p\right\rangle \neq 0, \quad\left\langle 0\left|J_{\mu_{1} \ldots \mu_{n}}(x)\right| p_{1}, p_{2}\right\rangle \neq 0
$$

Since $\sigma$ is the interpolating field for the Goldstone state, this means that at low energy $E \ll J^{1 / 2}$, the currents $J_{\mu_{1} \ldots \mu_{n}}(x)$ are

$$
J_{\mu_{1} \ldots \mu_{n}}(x)=A \partial_{\mu_{1}} \ldots \partial_{\mu_{n}} \sigma(x)+B \sigma(x) \stackrel{\leftrightarrow}{\partial}_{\mu_{1}} \ldots \stackrel{\leftrightarrow}{\partial}_{\mu_{n}} \sigma(x)
$$

The constants $A$ and $B$ can be extracted from Eqs. (3840). Even before any computation, $1 / N$ counting gives

$$
A=\mathcal{O}(1 / N), \quad B=\mathcal{O}\left(1 / N^{2}\right)
$$

In conclusion, when $g_{6}=g_{c}$, at any point outside the origin of the moduli space, conformal invariance is broken by the $\mathrm{VEV}$ of $\sigma$. At low energy $E \ll\langle\sigma\rangle$, the only light propagating degree of freedom is the Goldstone boson $\sigma$ itself. The dual interpretation of this phenomenon in the bulk is that the $4 \mathrm{~d}$ space flows from an AdS with curvature radius $R$ to another AdS space with radius $R^{\prime} \ll R$. Such flows perhaps may be described by bulk duals of the CFT, in analogy to what was done in $A d S_{5}$ in [19].

It is instructive to apply the formalism developed in this section to a cubic deformation of the IR limit of SYM with 16 supercharges in 3d. In this case, we have a good control of the bulk dual, while the boundary theory is hard to study directly. 


\section{More on $A d S_{4}$ Supergravity Duals of 3d CFTs and their Instabilities}

We spent some time in analysing the $O(N)$ model because while it is very simple as a boundary theory, yet it may shed light on a quite mysterious bulk theory on $A d S_{4}$. If we want to gain more control on the bulk theory, instead, we should look elsewhere. For instance, we could take as CFT the IR limit of a 3d super Yang-Mills $S U(N)$ theory with 16 supercharges in the large $N$ limit. Its holographic dual is the low-energy limit of a theory living on a stack of M-theory branes, which is $11 \mathrm{~d}$ supergravity compactified on $A d S_{4} \times S_{7}$ [20]. The dual theory describing the correlators of the superconformal multiplet containing the $3 \mathrm{~d}$ stress-energy tensor is a consistent dimensional reduction of such supergravity, namely, $N=8, S O(8)$ gauged supergravity [21]. The effect of multitrace deformations in this theory has been studied at length by Hertog and Horowtiz [11, 12, 13]. In particular, in [11, it was shown that a marginal, triple-trace deformation induces a big crunch singularity in the bulk. Correspondingly, the boundary CFT is unstable: the expectation value of the composite operator appearing in the deformation diverges in finite time. This result can be recovered straightforwardly using our formalism. First of all, we have to specialize our formulas to the deformation studied in [11]. The operator considered there has dimension $\Delta=1$, and it is dual to a scalar in $A d S_{4}$.

The unperturbed effective action for that operator, that we will call $F[\sigma]$ as in the previous section, is again largely determined by dimensional analysis. More precisely, when $\sigma$ is nonzero (and large), its kinetic term $K$ is

$$
K[\sigma]=\frac{N^{2}}{8}\left(\sigma^{-1} \partial_{\mu} \sigma \partial^{\mu} \sigma+\sigma\right) .
$$

Since the 3-d boundary CFT is strongly interacting, we cannot derive this result by writing down the action for the adjoint scalars $\phi$ of the super Yang-Mills theory, and using the fact that $\sigma \propto \operatorname{Tr} \phi^{2}$. We must use instead two properties: 1) the effective action is conformally invariant, and so is its kinetic term. 2) $\sigma$ has dimension $\Delta=1$. By setting $\sigma=\varphi^{2}$, Eq. (43) becomes the standard free action of a conformally coupled scalar $\varphi$ (not to be confused with $\phi !)$. The linear term in $\sigma$ arises because the boundary theory lives on $R \times S^{2}$, and the sphere has unit radius. Notice that the formula we wrote makes sense only when $\sigma^{2} \gg|\partial \sigma|$; in particular, it does not apply to the origin of the moduli space $(\sigma=0)$.

Let us add next the triple-trace perturbation $U=-N^{2}(f / 3) \sigma^{3}$. Using our general formulas, the effective action becomes $F+\int d^{3} x U$, so the perturbed potential is

$$
V(\sigma)=N^{2} \frac{f_{0}-f}{3} \sigma^{3}
$$


Here we have allowed the possibility that the unperturbed action has a (conformally invariant) potential $\left(f_{0} / 3\right) \sigma^{3}$, in analogy with the $O(N)$ case.

Consider now a cosmological solution as in [11, 12, 13, that depends only on time. The total energy is conserved so we can write a first integral of the equations of motion following from the Lagrangian $K+V^{5}$ :

$$
\frac{1}{8 \sigma}\left(\frac{d \sigma}{d t}\right)^{2}+\frac{\sigma}{8}+\frac{f_{0}-f}{3} \sigma^{3}=E .
$$

One particular solution, obtained by setting $E=0$, is $\sigma=\alpha / \cos t$. Substitution in the energy conservation equation gives

$$
\alpha \sin ^{2} t+\alpha \cos ^{2} t+\frac{8}{3}\left(f_{0}-f\right) \alpha^{3}=0,
$$

i.e. $\alpha=\sqrt{3 / 8\left(f-f_{0}\right)}$. So, when $f>f_{0}$ there exists a runaway solution in which the VEV $\sigma$ of a CFT operator diverges in finite time. The dual phenomenon in $A d S_{4}$ is that regular initial data evolve into a "big crunch" singularity that reaches the boundary in finite time [11. This is a clear cut case of singular behavior in the bulk that is not cured by embedding supergravity in a CFT dual.

This divergence may be less dramatic than it seems. In this case the proposed boundary theory is built out of adjoint fields. The boundary description of the bulk configuration is a three dimensional CFT with a non-supersymmetric yet marginal deformation of the form $\left(\operatorname{Tr} \phi^{2}\right)^{3}$. However, using the $O(N)$ vector model as a guide, we conjecture that such an operator is not marginal quantum mechanically, but is actually irrelevant ${ }^{6}$. So, we expect the classical deformation to become infrared irrelevant for any finite value of $N$.

This is a case in which stringy (i.e. $\mathcal{O}(1 / N)$ ) perturbative corrections come to the rescue, since an irrelevant operator signals large distance modifications of the AdS background. The IR divergence makes the smooth initial condition leading to a big crunch ill defined, at least in the sense that it has infinite energy with respect to an asymptotically AdS background.

This is not the type of singularity that string theory on AdS is supposed to resolve because at finite $N$ the initial data leading to a big crunch instability are not well defined near the boundary of $A d S_{4}$. The dual statement is that in the presence of a runaway solution where

\footnotetext{
${ }^{5}$ Recall that we use the mostly plus convention for the Lorentzian metric.

${ }^{6}$ In the adjoint $N=16 \mathrm{CFT}$, the triple-trace deformation mixes at $\mathcal{O}(1 / N)$ with single-trace operators. Since the IR of the deformed theory is non supersymmetric, the triple-trace will generically mix with all operators with the same unbroken global symmetries. These will include irrelevant operators that dominate the OPE at short-distance. In the holographic dual, they also determine the near-boundary behavior of the metric.
} 
some of the VEVs diverge in finite time, the CFT needs a UV completion. Moreover, the latetime behavior of the CFT depends strongly on that completion and is therefore non-universal. The simplest way to understand this is to notice that in order to define the CFT, one needs to introduce a UV cutoff $\Lambda$, even when the theory is finite ${ }^{7}$.

In the case of strings/M-theory, this UV cutoff is quite physical: it is the string/M-theory mass scale. The effect of the cutoff manifests itself in $F[\sigma]$ through higher-dimensional operators, weighted by inverse powers of $\Lambda$. Thus, the complete potential of $V$ is not as in Eq. (44); rather, it schematically reads

$$
V(\sigma)=N^{2} \frac{f_{0}-f}{3} \sigma^{3}+\sum_{n=4}^{\infty} c_{n} \Lambda^{3-n} \sigma^{n}
$$

The coefficients $c_{n}$ can be of order $N^{2}$ or less. In any case, the effect of the higher-dimensional operators cannot be neglected when $\sigma$ is so large that for some $n N^{2}\left(f_{0}-f\right) \sigma^{3} \sim c_{n} \Lambda^{3-n} \sigma^{n}$.

The system needs to be modified in the UV and in that case one simply does not know what really does happen at late times when the singularity approaches the boundary.

Finally, we should point out that in some cases the UV modification can be quite simple and involve no exotic physics. It could be as simple as an extra massive degree of freedom.

To see that, let us consider again the deformed $O(N)$ model we studied in sections 2 and 3 and add to it a single massive scalar $S$. The Lagrangian density is

$$
L=\frac{N}{2}\left[\partial_{m} \phi \partial^{m} \boldsymbol{\phi}+\partial_{m} S \partial^{m} S+\alpha M^{3 / 2} \phi^{2} S+\frac{1}{2} M \phi^{2} S^{2}+\frac{\beta}{2} M\left(\phi^{2}\right)^{2}+\frac{\gamma}{2} M^{2} S^{2}\right] .
$$

Here $M$ is a mass parameter and $\alpha, \beta, \gamma$ are arbitrary, positive, dimensionless constants. The potential is obviously bounded below.

At energy scales $E \ll M$ we can integrate out the field $S$ using its equations of motion. The contribution from the kinetic term is negligible so we have

$$
\alpha M^{3 / 2} \phi^{2}+\left(M \phi^{2}+\gamma M^{2}\right) S=0 .
$$

Substituting into eq. (48) we get a potential for $\phi$

$$
V=N\left[\frac{\beta}{2} M\left(\phi^{2}\right)^{2}-\frac{\alpha^{2} M^{3}\left(\phi^{2}\right)^{2}}{2 M \phi^{2}+2 \gamma M^{2}}\right]
$$

The term proportional to $\left(\phi^{2}\right)^{2}$ in this potential can be canceled by setting $\alpha^{2} / \gamma=\beta$. Then, the potential starts with the dimension-3 operator $\left(\phi^{2}\right)^{3}$. Precisely:

$$
V=N\left[-\frac{\alpha^{2} M}{2 \gamma}\left(\phi^{2}\right)^{2} \sum_{n=1}^{\infty}\left(-\frac{\phi^{2}}{\gamma M}\right)^{n}\right]
$$

\footnotetext{
${ }^{7}$ A simple example is the $O(N)$ model when $g_{6}$ is larger than the critical value. There, a careful treatment of the theory already at $N=\infty$ shows that in the presence of a cutoff all masses are of the order of the cutoff, so that the theory does not possess a universal low energy limit.
} 
Define $g=\alpha^{2} / 2 \gamma^{2}$. Then, in the limit $\gamma \rightarrow \infty, \alpha \rightarrow \infty, g=$ constant, all irrelevant terms vanish as inverse powers of $\gamma$.

Notice that the UV complete theory is always stable, even when $g>g_{c}$. The UV completion is recovered by "integrating in" the field $S$. Unlike the case of $N=1$ superpotentials, here the procedure is highly non unique.

This toy example suggests us also an alternative description of the resolution of the singularity in the adjoint model. In that case, the UV theory is most likely strongly interacting. Thus, terms which may seem unstable when extrapolated from weak coupling could actually represent a stable potential, when evaluated appropriately at strong coupling around a UV fixed point. So, the large- $\phi^{2}$ instability cannot be used as a sure diagnostic to invalidate the theory.

\section{Discussion/Conclusions}

We conclude with some general remarks on the meaning of instabilities occurring both in the bulk and in the dual boundary CFT, and whether string theory should cure those instabilities. The argument could be posed in the following manner. Assume one is somehow given a bulk theory containing a singularity and its corresponding CFT boundary dual, also singular or unstable. What is the interpretation of the instability in a complete string theoretical picture?

One may think that an instability of the boundary CFT is a disaster. In fact one can imagine instabilities in several forms. They could correspond to non-positive definite, relevant operators in the boundary theory which destabilize a flat direction. They could be marginal unstable deformations, and they could also come in the form of irrelevant, unstable boundary operators. The relevant and marginal operators need to be protected so that they do not become irrelevant as the coupling increases. All these cases have in common the feature that they indeed destabilize the boundary theory. Moreover, such deformations correspond to introducing various forms of repulsive forces among the branes whose near-horizon limit generated the bulk geometry. Such configurations will be unstable in the bulk and the branes will search for a new equilibrium configuration. Generically they may do so by fleeing to infinity, perhaps flat 10d space. These are indeed unstable configurations $a b$ initio and it is not one of the tasks of string theory on AdS to resolve them. Differently said, these pathologies signal instabilities of the near-horizon limit of string theory, not of string theory per se. [22, 23, 24].

If we examine more closely the different types of instabilities we find that they manifest themselves in the following manner. In the relevant case, the instability may emerge at some time, say $t=0$, deep inside the bulk and then spread out to the boundary along a null geodesic. On the other hand an irrelevant boundary instability generates already from the "start" at $t=0$ 
a greatly deformed bulk metric near the boundary. This is the particular case we have discussed in this note and it corresponds to an unstable bulk configuration which string theory need not resolve. This observation still leaves open the question of what would be the appropriate diagnostic for a real failure of non-perturbative string theory to resolve a singularity. For the time being, all instabilities exhibited in the literature signal at most a sickness of the nearhorizon limit. On the other hand, once a stable CFT dual to an AdS background exists, then one knows that all bulk singularities are indeed resolved. For instance, the unstable deformation of the $O(N)$ model at $g_{6}>g_{c}$ can be stabilised by the simple UV completion we described at the end of last section. Once this is done, the bulk singularity no longer reaches the boundary. The same phenomenon happens in the CFT dual to $11 \mathrm{~d}$ supergravity on $A d S_{4} \times S_{7}$ we studied in section 5: when the unstable potential is stabilised by irrelevant operators, the singularity does not hit the boundary but evolves instead into a giant black hole [13].

Finally we may ask ourselves if there exist universal properties shared by all resolutions of the instabilities of the low-energy sector of the dual boundary field theory. When the instability is resolved by introducing irrelevant operators the theory may seem to lose its predictive power. On the other hand there is a possible universal signature of stabilization: a boundary theory consisting of one or several stationary points, stable as well as unstable, may enable one to resolve a cosmological singularity. The story may go always as follows: near the unstable extrema of the potential, the bulk theory will effectively describe a big bang or big crunch, but that potentially singular behavior will eventually change into another one, which in the boundary theory will be described as a relaxation towards one of its true stable minima.

\section{Acknowledgments}

We thank O. Aharony, I. Klebanov, H. Neuberger and A. Polyakov for useful discussions. M.P. and E.R. would like to thank the KITP at UCSB for hospitality. S.E., A.G. and M.P. would like to thank CERN for hospitality. This work is supported in part by the American-Israeli Bi-National Science Foundation, the Israel Research Foundation, the European Union grant MRTN-CT-2004-512194, and DOE grant DE-FG02-90ER40560. M.P. is supported in part by NSF grant PHY-0245068, and by a Marie Curie chair, contract MEXC-CT-2003-509748 (SAG@SNS). 


\section{References}

[1] E. S. Fradkin and M. A. Vasiliev, Nucl. Phys. B 291, 141 (1987); Phys. Lett. B 189, 89 (1987);

[2] M. A. Vasiliev, Comptes Rendus Physique 5, 1101 (2004) arXiv:hep-th/0409260.

[3] I. R. Klebanov and A. M. Polyakov, Phys. Lett. B 550, 213 (2002) arXiv:hep-th/0210114.

[4] L. Girardello, M. Porrati and A. Zaffaroni, Phys. Lett. B 561, 289 (2003) arXiv:hep-th/0212181.

[5] W. A. Bardeen, M. Moshe and M. Bander, Phys. Rev. Lett. 52, 1188 (1984).

[6] J. L. F. Barbon and C. Hoyos, JHEP 0401, 049 (2004) arXiv:hep-th/0311274.

[7] W. A. Bardeen, K. Higashijima and M. Moshe, Nucl. Phys. B 250, 437 (1985).

[8] D. S. Berman and E. Rabinovici, arXiv:hep-th/0210044.

[9] E. Rabinovici, B. Saering and W. A. Bardeen, Phys. Rev. D 36 (1987) 562.

[10] T. Banks, private communication.

[11] T. Hertog and G. T. Horowitz, JHEP 0407, 073 (2004) arXiv:hep-th/0406134.

[12] T. Hertog and G. T. Horowitz, Phys. Rev. Lett. 94, 221301 (2005) arXiv:hep-th/0412169.

[13] T. Hertog and G. T. Horowitz, JHEP 0504, 005 (2005) arXiv:hep-th/0503071.

[14] E. Witten, arXiv:hep-th/0112258.

[15] M. Berkooz, A. Sever and A. Shomer, JHEP 0205, 034 (2002) arXiv:hep-th/0112264;

A. Sever and A. Shomer, JHEP 0207, 027 (2002) arXiv:hep-th/0203168.

[16] D. J. Amit and E. Rabinovici, Nucl. Phys. B 257, 371 (1985).

[17] W. Muck, Phys. Lett. B 531, 301 (2002) arXiv:hep-th/0201100.

[18] S. S. Gubser and I. R. Klebanov, arXiv:hep-th/0212138.

[19] L. Girardello, M. Petrini, M. Porrati and A. Zaffaroni, JHEP 9812, 022 (1998) arXiv:hep-th/9810126. 
[20] J. M. Maldacena, Adv. Theor. Math. Phys. 2, 231 (1998) [Int. J. Theor. Phys. 38, 1113 (1999)] arXiv:hep-th/9711200.

[21] B. de Wit and H. Nicolai, Nucl. Phys. B 208, 323 (1982).

[22] N. Seiberg and E. Witten, JHEP 9904, 017 (1999) arXiv:hep-th/9903224.

[23] J. Maldacena and L. Maoz, JHEP 0402, 053 (2004) arXiv:hep-th/0401024.

[24] M. Kleban, M. Porrati and R. Rabadan, JHEP 0508, 016 (2005) arXiv:hep-th/0409242. 